\title{
A FUZZY BEHAVIORIST APPROACH TO SENSOR-BASED ROBOT CONTROL*
}

\author{
François G. Pin \\ Robotics and Process Systems Division \\ Oak Ridge National Laboratory \\ P.O. Box 2008 \\ Oak Ridge, TN 37831-6305
}

\begin{abstract}
"The submitted manuscript has been
authored by a contractor of the U.S. Government under contract No. DEAC05-960R22464. Accordingly, the U.S.

Government retains a nonexciusive, royalty-free license to publish or reproduce the published form of this contribution, or allow others to do so, for U.S. Government purposes."
\end{abstract}

Contact: Dr. François G. Pin, Oak Ridge National Laboratory, P.O. Box 2008, Oak Ridge, TN 37831-6305, Telephone: (423)574-6130, Fax: (423)574-4624, E-mail: pin@ornl.gov

To Appear in: Proceedings of ISMIS '96, the Ninth International Symposium on Methodologies for Intelligent Systems, Zakopane, Poland, June 10-13, 1996

Also in "Lecture Notes for Artificial Intelligence," Springer-Verlag, 1996

*Oak Ridge National Laboratory, managed by Lockheed Martin Energy Research Corp. for the U.S. Department of Energy under contract number DE-AC05-96OR22464. 


\section{DISCLAIMER}

Portions of this document may be illegible in electronic image products. Images are produced from the best available original document. 


\title{
A FUZZY BEHAVIORIST APPROACH TO SENSOR-BASED ROBOT CONTROL
}

\author{
François G. Pin \\ Robotics and Process Systems Division \\ Oak Ridge National Laboratory \\ P.O. Box 2008 \\ Oak Ridge, TN 37831-6305 \\ Telephone: (423)574-6130, Fax: (423)574-4624, E-mail: pin@ornl.gov
}

\begin{abstract}
Sensor-based operation of autonomous robots in unstructured and/or outdoor environments has revealed to be an extremely chalienging problem. mainly because of the difficulties encountered when attempting to represent the many uncertainties which are always present in the real world. These uncertainties are primarily due to sensor imprecisions and unpredictability of the environment, i.e., lack of full knowledge of the environment characteristics and dynamics. An approach, which we have named the "Fuzzy Behaviorist Approach" (FBA) is proposed in an attempt to remedy some of these difficulties. This approach is based on the representation of the system's uncertainties using Fuzzy Set Theory-based approximations and on the representation of the reasoning and control schemes as sets of elemental behaviors. Using the FBA, a formalism for rule base development and an automated generator of fuzzy rules have been developed. This automated system can automatically construct the set of membership functions corresponding to fuzzy behaviors. once these have been expressed in qualitative terms by the user. The system also checks for completeness of the rule base and for non-redundancy of the rules (which has traditionally been a major hurdle in rule base development). Two major conceptual features. the suppression and inhibition mechanisms which allow to express a dominance between behaviors are discussed in detail. Some experimental results obtained with the automated fuzz: rule generator applied to the domain of sensor-based navigation in a priori unknown environments, using one of our autonomous test-bed robots as well as a real car in outdoor environments. are then reviewed and discussed to illustrate the feasibility of large-scale automatic fuzzy rule generation using the "Fuzzy Behaviorist" concepts.
\end{abstract}

\section{INTRODUCTION}

A significant research activity has taken place over the past two decades in the area of autonomous mobile robot navigation. The paramount complexity of the sensorbased navigation problem in unstructured environments arises mainly from the uncertainties which exist and become pervasive in the overall system (which includes the robot and its surrounding domain). In addition to the typical sensor inaccuracies (there are no such things as "perfect" sensors), the dynamics and unpredictability of the environment generate very large uncertainties in the perception and reasoning systems, i.e. it becomes impossible to generate complete or exact models of the system (robot and environment) and or of its behavior. These uncertainties, in tum, typically propagate through the control systems and lead to further inaccuracies or errors (e.g., in the robot position. in the sensor orientation) which compound the problem by increasing the uncertainties on the perception. In these conditions, the overall cost (time. computational needs. computing resources. etc.) of achieving the type of precision which was common in structured and static environments jumps by

DISCLAIMER

This report was prepared as an account of work sponsored by an agency of the United States Government. Neither the United States Government nor any agency thereof, nor any of their employees, makes any warranty, express or implied, or assumes any legal liability or responsibility for the accuracy, completeness, or usefulness of any information, apparatus, product, or process disclosed, or represents that its use would not infringe privately owned rights. Reference herein to any specific commercial product; process, or service by trade name, trademark, manufacturer, or otherwise does not necessarily constitute or imply its endorsement, recommendation, or favoring by the United States Government or any agency thereof. The views and opinions of authors expressed herein do not necessarily state or reflect those of the United States Government or any agency thereof.

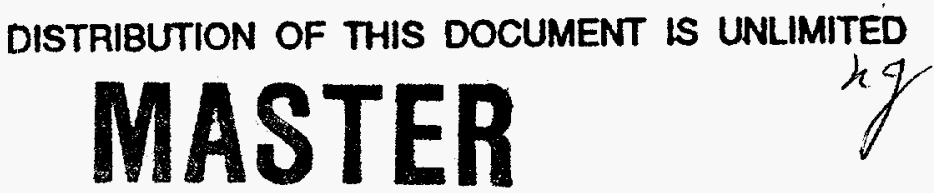


orders of magnitude, either from a requirement for much more refined sensors and perception data, or from the need for very time- and computation-expensive methods such as uncertainty analysis and propagation techniques. The impact of this cost increase is particularly important for real-time systems (where real-time is defined as the guarantee of producing a response within a prescribed amount of time), which become much more difficult to design, if not impractical to implement in realistic situations.

Humans on the other hand, seem to handle/cope very well with uncertain and unpredictable environments, often relying on approximate or qualitative data and reasoning to make decisions and to successfully accomplish their objectives. Several approximate reasoning theories and associated mathematical algebra have been developed over the past two decades (e.g., see methods and references in [1]), the most commonly used today for applications to control systems being Zadeh's Theory of Fuzzy Sets [2]-[4]. This theory is at the basis of very successful implementations varying from control of subway cars, elevators, cement kilns, washing machines, cameras and camcorders, inverted pendulums, to painting processes and color image reconstruction, to even Ping-Pong playing robots (e.g., see [1], [5], [6]). One of the important factors which have prevented the wide-spread utilization of approximate reasoning methodologies in real-time systems has been the lack of computer hardware allowing processing and inferencing directly in terms of approximate or linguistic, or "fuzzy" variables (e.g., far, fast, slow, left, faster) and approximate rules (e.g., if obstacle is close, then go slower; if temperature is high and pressure is increasing, then decrease power a lot). Prospective implementations thus had to rely on simulations of the approximate reasoning schemes on conventional computers based on "crisp" processing. The result was a significant penalty in speed of operation, typically prohibiting applications in most "hard real-time" systems. Over the last half decade, however, several innovations have allowed some bridging of this gap; in particular, unique computer boards have recently been developed which use customdesigned VLSI Fuzzy Inferencing chips (e.g., see [7], [8], and [9]) on VME-bus compatible boards. These systems can be directly programmed in terms of qualitative variables and rules and, when incorporated in a control system, can directly communicate and interface with robotic hardware (e.g., with motors, actuators). Such computer hardware developments have proven extremely useful in supporting the developments needed in the area of approximate reasoning for real-time "intelligent" machines, and have been a strong basis [9],[10] for the activities reported here.

\section{FUZZY BEHAVIORIST APPROACH AND RULE GENERATION FOR SENSOR-BASED CONTROL}

The problem of autonomous mobile robots navigating in a priori unknown and unpredictable environments was selected as a paradigm for research on qualitative reasoning and control systems because its characteristics rank very high on the list of criteria that typically indicate suitability of a reasoning problem for representation using qualitative logic and resolution using trade-offs.

- The input to the control system, particularly when provided by sonar range finders and odometric wheel encoders, is inaccurate, sparse, uncertain and/or unreliable.

- No complete mathematical representation exists of the process termed "navigation" although, as demonstrated by humans, a logic for this process exists which can typically be represented and successfully processed in terms of linguistic variables. 
- The approximations involved in the numerical representation of the system and its environment (e.g., geometric representations, map discretization in grid) are significant.

- By its given nature the behavior of an outdoor environment is unpredictable, leading to large uncertainties in its representation and frequent need for trade-off of speed vs. precision.

Several research groups have studied approximate reasoning techniques, in particular fuzzy logic, to mimic human reasoning capabilities in navigation tasks (e.g., see [10] to [13]). In all these applications, the sensor-based decision-making process has been implemented as a set of fuzzy rules which, together, express the desired navigation decisions of the robot for various combinations of the input data. Very successful results have been achieved when the number and complexity of the rules were small. When these increased, however, and/or the perception system grew more sophisticated (i.e., more sensory input data is provided), the typical difficulties encountered with large rule base systems emerged: the lack of established formalism for the development of rule bases - in particular with respect to completeness, interaction, and redundancy of the rules - made the actual coding of the fuzzy rules an iterative empirical process, requiring lengthy trial-and-error experiments.

\subsection{Fuzzy Behaviorist Approach}

In an attempt to alleviate this general shortcoming of rule-base system development, we recently proposed a "Fuzzy Behaviorist Approach" (FBA) which provides a formalism for the development of fuzzy rule systems for control of autonomous robots. The basic premises underlying our proposed FBA are as follows: .

- Each action of the robot results from the concatenation of elemental behaviors.

- Each elemental behavior is a direct mapping from a single stimulus mode to a single output control.

- Each behavior is represented by one or a set of fuzzy rules which are defined by the membership functions of the rule's antecedent (stimuli) and consequence (output controls).

- Each mode of stimulus corresponds to a single dimension of the input space and is independent, in a possibilistic framework, of other stimulus modes.

- For behaviors effecting the same output control dimension, the possible conflicts between behaviors with stimuli that overlap in the multidimensional input space must be resolved through the expression of the respective dominance between the various behaviors.

- Each type of input data provided by the sensors is fuzzified with a membership function expressing, as a possibility distribution, the uncertainty associated with the specific measurement or calculation (see [10] or [14] for a detailed discussion of this process). The inferencing laws of the Fuzzy Sets Theory are then used to embody the rule-based reasoning.

Cast in the framework of Fuzzy Set Theory, these principles can be expressed as follows: the inferencing, $l$, of the (robot) reasoning system provides the relationship between the input universe of discourse $U$ and the output universe of discourse $V$. The input universe of discourse $U$ is multidimensional, with each dimension representing a type of input data on which the inferencing can act (e.g., distance to obstacle to the right of route, direction to the goal, distance to the goal), i.e., each 
input dimension is a mode of stimulus, $s_{i}$, that can excite the inferencing. Similarly, the output universe of discourse $V$, is multidimensional, with each dimension representing a type of output data, i.e., a type of control, $c_{j}$, (e.g., turn control, motor \#1 speed) that can be implemented. Thus we have:

$$
I: U\left(s_{1}, s_{2}, \ldots, s_{i}, \ldots, s_{n}\right) \rightarrow V\left(c_{1}, c_{2}, \ldots, c_{j}, \ldots, c_{m}\right)
$$

The total numbers of possible stimulus modes, $n$, and of control modes, $m$, are of course dependent on the sensory and actuation capabilities implemented on the robot. Each dimension $s_{i}$ or $c_{j}$ is a one-dimensional space on which fuzzy sets can be defined using membership functions in the conventional manner (e.g., see [2], [3], [4], and [5]). An elemental behavior, $B_{i j}$, is thus defined as a direct mapping from $s_{i}$ to $c_{j}$ :

$$
B_{i j}: s_{i} \rightarrow c_{j},
$$

which is represented by one or several fuzzy rules relating fuzzy subsets of $s_{i}$ to fuzzy subsets of $c_{j}$. As an example, assume $s_{3}$ represents the "direction to the goal" input dimension and $c_{2}$ represents the "turn control" output dimension, then the behavior $B_{32}$, "turn control as a function of the goal direction," would include fuzzy rules of the type: IF (direction to the goal is left) THEN (turn value is positive), where left and positive are fuzzy subsets defined by their membership functions on $s_{3}$ and $c_{2}$, respectively.

Note that the fourth requirement of the approach specifies that the input space be designed such that the stimulus modes (input dimensions), $s_{i}$, are independent of each other, i.e., such that the possibility for the ith input to be any fuzzy subset in $s_{i}$ is completely independent of the possibility for any other input to be any fuzzy subset on their stimulus mode. In other words, the possibilities for any and all stimuli to occur are unrelated and independent of each other. This allows a behavior $B_{i j}$ to be extended to a mapping $B_{i}^{*}$, from $U$ to $V$ as

$$
B_{i j}^{*}: U\left(\#, \#, \#, \ldots, s_{1}, \# \ldots, \#\right) \rightarrow V\left(\phi, \phi, \ldots c_{j}, \phi, \ldots, \phi\right),
$$

where the \# and $\phi$ signs represent "non-significant" input and output dimensions, respectively. By definition, $s_{1}$ and $c$, are the "significant" input and output dimensions of behavior $B_{i j}^{*}$. For example, the behavior $B_{32}$ discussed above has 3 (for input) and 2 (for output) as significant dimensions and would be extended to a behavior $B_{32}^{*}$ on the multidimensional input and output spaces with fuzzy rules now expressed as:

IF (input 1 is anything and input 2 is anything and direction to the goal is left and input 4 is anything and ...) THEN (output 1 is do nothing and turn value is positive and output 3 is do nothing and ...) 
and the fuzzy subsets anything and do nothing have membership functions uniformly equal to 1 and to 0 over the entire range of their respective input and output dimensions.

With these features, rule bases embodying sets of elemental fuzzy behaviors can be very easily generated (e.g., see [9] and [10]). They can also be readily augmented with additional behaviors, either to handle situations of increasing complexity (e.g., see [10] and [14]) or if additional perception or control capabilities are added to the system, requiring larger dimensions of the input or output spaces. In these cases, however, a very important aspect of the formalism which needs to be emphasized here is the requirement for independence and non-conflict of the stimuli of the behaviors effecting the same output controls. This requirement simply expresses that only one action command can be sent to a single output control for any given stimulus (a single point in the input space). This leads to newly added (or acquired) behaviors having to "dominate," or "be dominated by," some of the existing behaviors in one or more regions of the input space. This concept of dominance between behaviors, which exhibits itself in almost every action of our everyday life, has been illustrated in previous papers [10], [14], with the major point that an indication of what behavior dominates the other in what region of their overlapping areas in the input space, must be specified into the system if two "seemingly conflicting" behaviors are to produce a reasonable advanced behavior. In the automated system, this is accomplished through the "suppression" mechanism, in which the output membership function of the dominant behavior is modified, so that its weight will appropriately overpower that of the other behavior in the c.g. calculation. If due to previous dominances and/or suppression requirements with respect to other behaviors, the membership function can not be made adequately overpowering. then the system uses the concept of "inhibition" to express the behavior's dominance. Applying inhibition within the overlapping region of input space consists in partially truncating the input membership function of the "weaker" behavior so that the dominant behavior always triggers with a greater strength. Although both suppression and inhibition mechanisms result in expressing the desired dominance, their concepts are quite different: one basically operates on the relative weight of the behaviors in the output space while the other modifies the triggering conditions of the behaviors in the input space. Figure 1 schematically shows these very intuitive suppression and inhibition mechanisms on a simple example in which two rules, each with two input and one output, act on the speed control of a robot. The first rule, which may be part of a behavior for speed control as a function of frontal obstacle proximity, states that if the obstacle is very close. then the speed should be very small. The second rule states that if the goal is straight ahead then the speed is fast. The membership functions uniformly equal to 1 over their entire range represent the non-significant input dimensions of each behavior.

\subsection{Automated System}

Just like different people may use different strategies, different rules, and different qualitative variables to express their navigation process, and still navigate efficiently "in their own way," several strategies may be used to embody a particular process in a rule base, i.e., there is not a single or unique rule base representation of a given process. In the automated system. the user inputs the strategy for the rules in a "qualitative" form using the format shown in Fig. 2. 


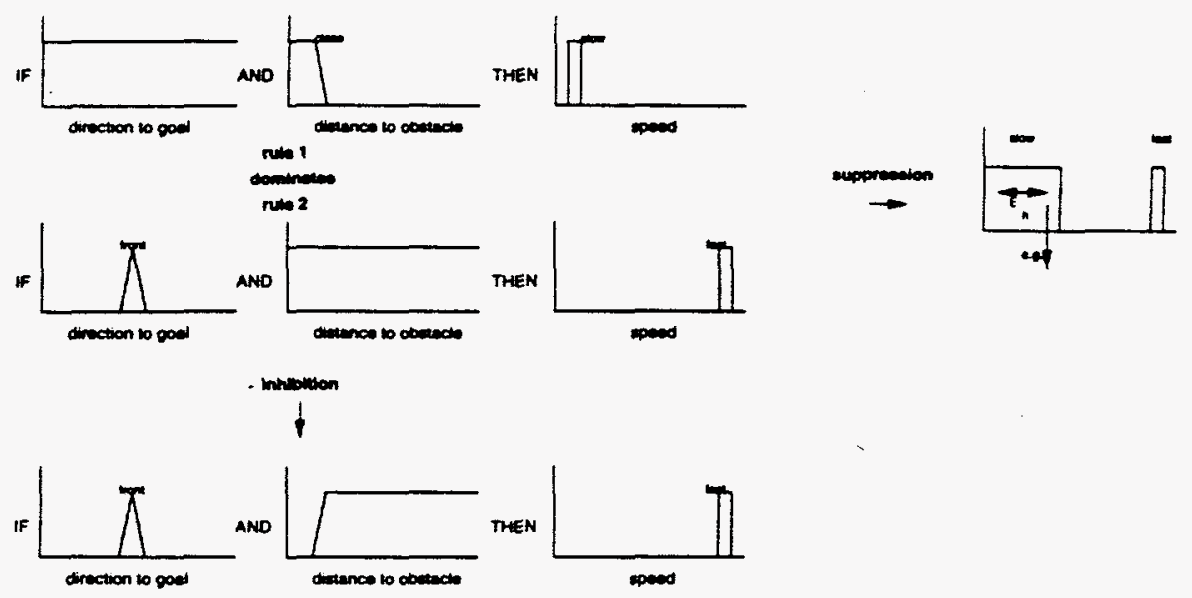

Fig. 1. Schematic of the suppression and inhibition mechanisms.

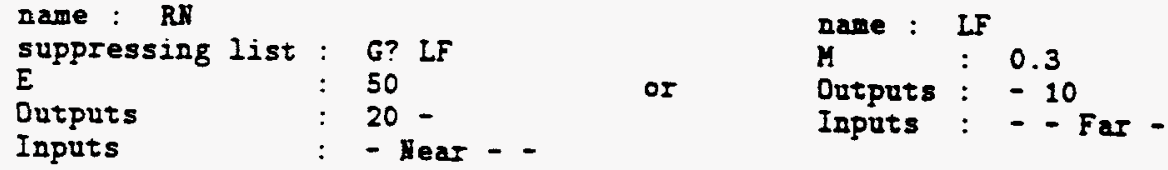

Fig. 2. Input format for the automated rule generation system.

The five-line format in Fig. 2 describes one rule, with the first line giving the "reference name" of the rule. the second line listing the names of the rules or behaviors which are suppressed (or inhibited) by this rule, the third line giving the suppression parameter $E$ (which will be described in the following paragraphs), and the last two lines specifying what the desired input and output of the rule on the "significant" (see Section 2.1) input and output dimensions of the behavior are. In the current version of the automated system. each rule is assumed to be of the form

IF ( $A$ is $A_{1}$ and $B$ is $B_{1}$ and $C$ is $C_{1}$ and $D$ is $D_{1}$ ) THEN ( $E$ is $E_{1}$ and $F$ is $F_{1}$ ), (4)

therefore operating on four input and two output channels. Although extension to any number of input and output channels is possible, this configuration was chosen in the initial version of the automated system because it corresponds to what is available on the custom-designed VLSI fuzzy inferencing chips and boards (see [7], [8], and [9]) which we utilize in our experimental work. Use of the character "?" in the suppression list of line 2. such as "G?" in the example of Fig. 2, indicates that the rule suppresses all other rules whose name includes the other character, in this case all rules whose name begins with the letter "G." If the subject rule does not suppress other rules, then the first character of line 2 is the letter $M$ followed by a number expressing the desired weight $m$ of the output (explained in the following paragraphs), and the line giving the suppression parameter $E$ is omitted. The line labeled "outputs" in Fig. 2 indicates which of the two outputs is effected by the behavior, with the number, expressed in percent of the scaled output range, specifying the 
desired center of mass of the membership function of the output ( $E_{1}$ or $F_{1}$ in Eq. (4)). The line labeled "inputs" specifies which one of the four input channels is the behavior's "significant" input dimension, and the qualitative name indicates which fuzzy set constitutes the antecedent $\left(A_{1}, B_{1}, C_{1}\right.$, or $D_{1}$ in Eq. (4)) of the rule on the input dimension. The membership functions defining the input related fuzzy sets are defined by the user on a behavior-by-behavior basis (e.g., see [8] or [9]), and can be stored according to their "name" in a "membership function library."

When the user has listed all the rules of the desired behaviors in the format of Fig. 2, the automated system can generate a "skeleton" of the rule base and check if it verifies the input-related requirements of the approach. In particular, the system constructs the four-dimensional input spaces for each of the two output dimensions, so that it can evaluate completeness of, and redundancy in, the rule base and report all instances to the user. For any region of incompleteness, i.e., regions of the input space not covered by any of the behaviors stimuli, the user decides on either the addition of a behavior to cover these possible stimuli, extension of the current behaviors (through extension of their input membership function) to include these input regions, or no modification if input data within these uncovered regions or "blind spots" are never expected to occur (for example if these regions correspond to values outside the operating range of the sensors). For the regions of redundancy, i.e., areas where stimuli from two or more behaviors are overlapping, the system reports every rule for which a dominance has not been specified but may be required because of the input overlapping. The user can then interactively add to or modify the dominance specifications in lines 2 and 3 of each rule, until all requirements of the approach are verified and all desired dominances are: expressed in the rule base. The actual generation of the rule base, including the suppression and/or inhibition mechanism, can then proceed as follows: initially, all rules are given a "standard" output membership function equal to 1 over a width of one bit, centered at the bit value expressed in line 4 of the "qualitative" expre:sion of the rule (see Fig. 2). The system checks the sets of rules that are effecting the same output dimension. If no suppression mechanism has been expressed between the rules because dominance is not necessary, then the output membership functions are unconstrained and they remain at their "standard" value. If a dominance his been expressed between two or more rules, then the dominant rule is the one that is modified if suppression is possible, otherwise the dominated rules are modified using inhibition. The automated process and the corresponding equations have been described in detail in [15].

\section{SAMPLE EXPERIMENTAL RESULTS}

Using the automated fuzzy rule generation system, a variety of rule bases were generated (e.g., see [10], [14]) for the sensor-based navigation of mobile robots in a priori unknown environments. The experiments involved both indoor and outdoor environments, and progressed from simple goal tracking navigation in laboratory-type situations to sensor-based driving of a car in frarking lot conditions, and from platforms with omnidirectional capabilities to robots with complex car-like kinematics including non-holonomic constraints. In what follows, sample results from some of these experiments are reviewed to illu strate the automatic rule generation process including the suppression and inhibition mechanisms. As discussed in detail in Refs. [13] and [14], the four sensory input to the fuzzy inferencing system are the goal direction (or target direction), which is updated at loop rate using the odometry 
system; and the minimum distances to obstacles obtained using groups of acoustic range finders in three $75^{\circ}$ wide sectors at the left, center, and right of the robot's travel direction. The two output of the inferencing system are the commands for turn increments and speed of the robot. Thus, navigation behaviors with these data can involve Goal Orientation (GO) and "front," "left," or "right" Obstacle Proximity (OP) as input, and Turn Control (TC) and Speed Control (SC) as output.

\subsection{Example of Basic Navigation Behaviors}

As mentioned previously, several rule bases, representing various "strategies," may be developed to solve a complex problem or to embody a complex behavioral process. Figure 3 shows plots of actual runs made with one of our small omnidirectional robots [16] to illustrate the overall reactive navigation obtained with the automatic generation of fuzzy rules. These plots are also given here to provide an example of the effect on the navigation behaviors which a dominance mechanism (suppression or inhibition) can produce. In the figures, the shaded areas represent the obstacles which were placed in the room, while the path of the robot is illustrated using the succession of circles showing the position of the robot every 20 loop rates. In Fig. 3-a, the rules of a basic rule base described in detail in [10] and [14] were used, which embody a very strong dominance of the obstacle avoidance rules over the goal tracking rules. Consequently, due to the almost constant proximity of the corridor walls, the suppression mechanism is quite effective in the early part of the run and the robot wanders around for quite a long time, guided principally by obstacle avoidance. It eventually gets positioned ideally to enter the corridor and then turns right, in a direction closest to the goal direction. It follows the corridor, and when reaching the end of the wall, turns left toward the goal. Clearly, the dominance of the obstacle avoidance rules over the "move to the goal" behavior may be too strong in this environment. For the sample run shown in Fig. 3-b, this dominance has been decreased, through an increase of the suppression parameter (see Figs. 1 and 2 , and [15]), and a corresponding rule base has been generated using the automated system. The robot is now seen to negotiate the entrance of the corridor much more rapidly because of the greater effect of the goal tracking behavior, resulting in a much shorter run to the goal. From an overall behavioral point-of-view, the simple change of the dominance of the "obstacle avoidance" over "move to the goal" behaviors in the rule base, has transformed the "shy" robot. driven mainly by obstacle avoidance in Fig. 3-a. into a "much braver" robot proceeding more rapidly toward its goal in Fig. 3-b. Note that this "transformation" has been accomplished without direct modification of the logic embodied in the elemental behaviors.

\subsection{Extension to Robots with Car-Like Kinematics}

One of the expected strengths of our proposed Fuzzy Behaviorist Approach using elemental "human-like" behaviors is that the linguistic logic embodied in the behaviors should be invariant across systems of similar characteristics. In other words, for robots with similar perceptive abilities and motion capabilities, the linguistic expression of given behaviors. and therefore their representation in the fuzzy framework. should be the same for compatible input and output. For example, a "goal tracking" behavior connecting the perceived goal direction to a rate of tum [e.g.. IF (goal is to the right) THEN (apply increment of tum to the right)] should be invariant for any robot which has a means to perceive the goal direction and to perform the required turn. 

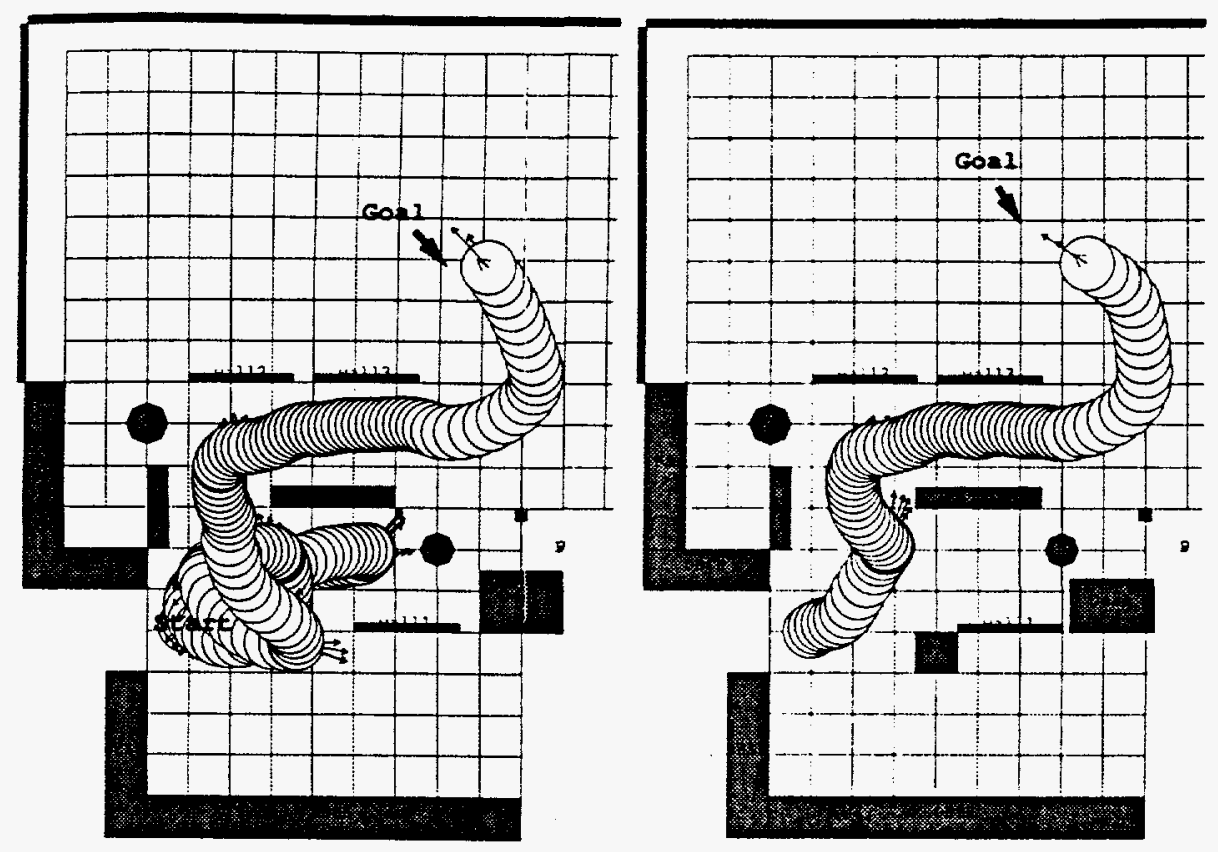

Fig. 3. Actual runs of the robot using automatically generated fuzzy rule bases with a) a strong behavioral dominance of obstacle avoidance over goal tracking, b) a lesser behavioral dominance of obstacle avoidance over goal tracking.

As a demonstration of this transportability of invariant behaviors from one system to another, the same behaviors and the same fuzcy rules that were utilized for the omnidirectional platform were used to implement the autonomous control of a car on the basis of the same three $75^{\circ}$ "wide and blurry sonar eyes" and goal direction input. To complete the navigation rule base for the driving of the car, a behavior had to be included to handle situations where "very near" obstacles are detected and the car does not have enough space to complete a tum away from obstacles because of its limited steering angle, therefore requiring some maneuvers using reverse gear. Another strength of our proposed Fuzzy Behaviorist Approach is its capability for superposition of elemental behaviors, allowing for progressive addition of behaviors to the system to resolve situations of increasing complexity

Both simulation and outdoor experiments were performed with the maneuvering behavior-augmenteed rule base and results can be found in Refs. [10] and [14]. Because of the unavailability of a car with automated actuation, no autonomous navigation tests were performed outdoors. However, the system was investigated for use as a "driver's aid" using one of the company cars. In the driver's aid mode, the same rule base, sensors, and inferencing system as in the previous experiments are used, however the commands produced by the rule base are displayed to the driver to guide him/her in driving the car. The driver conventionally uses the gas and brake pedals and steering wheel to implement the commands that are displayed on a portable computer screen located next to him/her in the cabin. From the system's 
development point of view, this inclusion of the human in the control chain effectively consisted in including a source of unpredictable noise and delays in the actuation system of the autonomous operation mode. The successful operation of the rule base in this mode of driving (see [19]) thus provided a stringent robustness test of the qualitative inferencing scheme and navigation system.

\section{CONCLUDING REMARKS}

An automated system to generate fuzzy rules from the qualitative description of a reasoning process has been developed. The automated system is built on the basis of the Fuzzy Behaviorist Approach which we proposed for the development of fuzzy rule bases embodying "human-like" behaviors in sensor-based decision-making systems. The concepts of suppression and inhibition of behaviors and the inclusion of corresponding mechanisms in the automated system have been described. Examples of the use of the automated system to generate fuzzy rule bases for the sensor-based navigation of autonomous robots have been discussed. Sample runs of the robots have been presented to illustrate the navigation behaviors obtained with the automatically generated fuzzy rule bases as well as the effect of a change in the interbehavior dominance expressed through the suppression and/or inhibition mechanism. Experiments with a real car have also been discussed to illustrate the capability of readily adding behaviors to the fuzzy rule base to resolve situations of increasing complexity and, as shown in the driver's aid feasibility study, the straightforward "linguistic" interfacing capability of the fuzzy behavior-based system. A variety of lessons and observations can be drawn from these experiments. Several of these, relevant to the topics dealt with here. are listed and/or discussed below.

- The entire navigation code or scheme consists of less than 20 fuzzy rules. Compared to the $30,000+$ lines of "crisp" coding which were previously utilized to accomplish the same task. the efficiency and gain in code development time, code and data storage space, etc., of the approach seems to be indisputable.

- Our observations showed the approximate reasoning scheme to be much more robust and reliable than the previously used "crisp" codes when faced with sensor inaccuracies and environmental uncertainties. This seems to clearly support the claim that, for situations where precision is not the primary goal, robotic tasks can be very efficiently accomplished using an approximate reasoning scheme, but also can be made more robust with respect to uncertainties through an implicit "folding" of these uncertainties within approximate variables and reasoning schemes.

- As illustrated through the various experiments involving small omnidirectional and indoor robots and/or an outdoor car. approximate reasoning schemes can be built to embody very generic functions. i.e.. they do not need to be system-specific. In other words, they can embody a reasoning strategy rather than a specific instantiation of the strategy. Funhermore. this genericness of the approximate reasoning schemes provides straight-fon ard transportability of codes among various systems with similar perception and motton means.

- A significant consequence of this genericness of approximate reasoning schemes is of course their scale up capability. As shown in the experiments, basic navigation schemes could be augmented and or enhanced with additional behaviors without rewriting the previously tested behaviors. The gain in development time resulting from this property of approximate reasoning schemes is expected to be substantial. 


\section{REFERENCES}

[1] Uncertainty in Artificial Intelligence, eds. L. N. Kanal and J. F. Lemmer, North-Holland (1988).

[2] Zadeh, L. A., "Fuzzy Set," Information and Control 8, 1965, pp. 338-353.

[3] Zadeh, L. A., "Outline of a New Approach to the Analysis of Complex Systems and Decision Processes," IEEE Transactions on Systems, Man, and Cybernetics SMC-3(1), 1973, pp. 28-45.

[4] Zadeh, L. A., "Fuzzy Logic," IEEE Computer 21(4), April 1988, pp. 83-93.

[5] Fuzzy Sets and Their Applications to Cognitive and Decision Processes, eds. L. A. Zadeh, K. S. Fu, K. Tanaka, and M. Shinamura, Academic Press, Inc., New York (1975).

[6] Industrial Applications of Fuzzy Control, ed. M. Sugeno, North-Holland (1985).

[7] Watanabe, H., W. Dettloff, and E. Yount, "A VLSI Fuzzy Logic Inference Engine for Real-Time Process Control," IEEE Journal of Solid State Circuits 25(2), 1990, pp. 376-382.

[8] Symon, J. R. and H. Watanabe, "Single Board System for Fuzzy Inference," in Proceedings of the Workshop on Software Tools for Distributed Intelligent Control Systems, September 1990, pp. 253-261.

[9] Pin, F. G., H. Watanabe, J. R. Symon, and R. S. Pattay, "Autonomous Navigation of a Mobile Robot Using Custom-Designed Qualitative Reasoning VLSI Chips and Boards," in Proceedings of the 1992 IEEE International Conference on Robotics and Automation, Nice, France, May 10-15, 1992, pp. 123-128.

[10] Pin, F. G. and Y. Watanabe, "Navigation of Mobile Robots Using a Fuzzy Behaviorist Approach and Custom-Designed Fuzzy Inferencing Boards," Robotica 12(6), 1994, pp. 491-503.

[11] Yen, J. and N. Pfluger, "A Fuzzy Logic Based Robot Navigation System," in Proceedings of the AAAI Symposium on Applications of Artificial Intelligence to Real-World Autonomous Mobile Robots, Cambridge, Massachusetts, October 23-25, 1992. pp. 195-199.

[12] Takeuchi, T. et al., "Fuzzy Control of a Mobile Robot for Obstacle Avoidance," Information Science 45, 1988, pp. 231-239.

[13] Sugeno, M. et al., "Fuzzy Algorithmic Control of Model Car by Oral Instructions," Fuszy Sets and Systems 32, 1989, pp. 207-219.

[14] Pin, F. G. and Y. Watanabe. "Steps Toward Sensor-Based Vehicle Navigation in Outdoor Environments Using a Fuzzy Behaviorist Approach," International Journal of Intelligent and Fuz:y. Systems 1(2), 1993, pp. 95-107.

[15] Pin, F. G. and Y. Watanabe. "Automatic Generation of Fuzzy Rules Using the Fuzzy Behaviorist Approach: The Case of Sensor-Based Robot Navigation," Intelligent Automation and Soft Computing 1(2), 1995. pp. 161-178.

[16] F. G. Pin and S. M. Killough, "A New Family of Omnidirectional and Holonomic Wheeled Platforms for Mobile Robots,," IEEE Transactions on Robotics and Automation 10(4), 1994. pp. 480-489. 